\title{
Average Link Stability with Energy-Aware Routing Protocol for MANETs
}

\author{
Sofian Hamad, Salem Belhaj \\ Northern Border University \\ Arar - Saudi Arabia
}

\author{
Muhana M. Muslam \\ Al-Imam Muhammad Ibn Saud Islamic University \\ Riyadh, Saudi Arabia
}

\begin{abstract}
This paper suggests the A-LSEA (Average Link Stability and Energy Aware) routing protocol for Mobile Ad-hoc Networks (MANETs). The main idea behind this algorithm is on the one hand, a node must have enough Residual Energy (RE) before retransmitting the Route Request (RREQ) and declaring itself as a participating node in the end-to-end path. On the other hand, the Link Life Time (LLT) between the sending node and the receiving node must be acceptable before transmitting the received RREQ. The combination of these two conditions provides more stability to the path and less frequent route breaks. The average results of the simulations collected from the suggested A-LSEA protocol showed a fairly significant improvement in the delivery ratio exceeding $10 \%$ and an increase in the network lifetime of approximately $20 \%$, compared to other re-active routing protocols.
\end{abstract}

Keywords-Mobile Ad-hoc Network (MANET); routing protocol; energy aware; link life time; $A O D V$

\section{INTRODUCTION AND MOTIVATION}

During normal operation of reactive routing protocols, the routing path between a source and a destination must be discovered before data packets transmission [1]. The routing process in MANETs requires that mobile nodes cooperate together to effectively direct traffic between communicating pairs [2]. The availability of the node is crucial for applying such cooperation. Indeed, its absence affects the state of active connections in its neighborhood. In MANETs, several factors can affect the availability of nodes and cause link breaks, such as interference, obstacles, mobility, and node residual energy (lifetime of the battery).

Two factors will be considered in this paper as being the main contributors to link breaks in MANETs, namely, 1) mobility; and 2) Remaining Energy (RE) of the mobile node.

Regarding the mobility of MANET nodes and the limitation of their power supplies, mobile nodes are considered as energy-constrained devices; this factor has an impact on the availability of the nodes as well as on the network lifetime. Besides, the routing control messages consume a significant amount of the node's battery [3]. Likewise, the mobility of nodes in MANETs is one of the main features of that cause frequent changes in the network topology and therefore increase the probability of link failures and route breaks. As a result, link failures cause the nodes to begin a path maintenance process to find alternate routes. Nevertheless, finding a new path requires a lot of bandwidth, consumes nodes batteries, and adversely effects on network performance by adding re-routing delays and routing overhead. Thus, the routing process should seek only the best routes ensuring long-term stability and sustainability, by taking into account nodes mobility and their residual energy.

The current research paper suggests a new path discovery algorithm using the RE of the nodes and their LLT. The main idea underlying the suggested algorithm is to transmit RREQ packets on stable links across nodes with sufficient RE and acceptable LLT among the participating nodes on the route. Thus, the suggested protocol can be deployed for MANETs with most existing on-demand routing schemes such as AODV [4], as well as DSR [5].

\section{STATE OF THE ART ON LINK STABILITY AND ENERGY AWARE PROTOCOLS}

In the MANETs literature, various LLT estimation methods already exist. Some of them are based on Received Signal Strength (RSS) [6], while other methods, predict the LLT using the location information of the nodes forming the links. Furthermore, several routing algorithms [7] exploit nodes RE and LLT as primary routing metrics to enable the selection of the best end-to-end (e2e) path for transmission, in terms of stability and energy saving.

In the following, the existing routing algorithms implementing the concept of RE and LLT will be discussed.

\section{A. RSS-Based Routing Protocols}

In [8], authors use Received Signal Strength (RSS) as the basic routing metric defining the quality of a link, which varies between two mobile nodes in accordance with a predefined Signal Strength Threshold (SST); it decreases when the RSS between the communicating nodes is lower than the predefined SST and increases in the opposite case.

Furthermore, the research paper [9] raises the Signal Stability-based Adaptive (SSA) routing protocol. In this method, the links are grouped according to the RSS metric. The route discovery mechanism consists on classifying neighbouring nodes connections into two groups: Weakly Connected (WC) and Strongly Connected (SC) links. This classification is carried out by the receiving nodes according to the RSS of the neighbouring nodes when they send the Route Request packet. During the transmission phase, SSA can go through WC links, causing path breaks.

The routing protocol based on the signal strength suggested in [10] first uses the previously established path for packet transfer. Subsequently, it modifies the established path to the strongest RSS. 
In [11], the authors proposed a local link management mechanism for OLSR [12]. They use a multi-layer mechanism based on RSS, which makes it possible to decide on the quality of the link; if it is improved or degraded. Moreover, The OLSR RFC [12] describes the hysteresis method dealing with packet loss. This technique anticipates the link breaks to strengthen link management and consequently improves the performance of the network.

\section{B. Locatio-Based Routing Protocols}

The Geographical Positioning System (GPS) [13] is used by most routing protocols using location to obtain motion information about nodes in the network such as direction, coordinates, and speed. In [14], the authors propose a route lifetime and a link prediction algorithm based on node location and motion information. They supposed that all the clocks of the network nodes are synchronized with the GPS clock itself. Thus, the connection time between a couple of nodes can be calculated using (1), if the motion parameters of the two connected nodes are known (direction, coordinates and speed). The idea used in [14] is to estimate the Link Expiration Time (LET) of the path at every hop, which makes it possible to estimate the e2e Route Expiration Time (RET), defined as the minimum LET of the links concerned in an e2e path. Then the route with the highest RET is chosen as the best path.

$$
L E T=\frac{-(x+v)+\sqrt{\left(x^{2}+y^{2}\right) r^{2}-(\mathrm{xz}-\mathrm{vy})}}{x^{2}+y^{2}}
$$

Where,

$$
\begin{aligned}
& x=a_{i} \cos \theta_{i}-a_{j} \cos \theta_{j}, \\
& v=b_{i}-b_{j} \\
& y=a_{i} \sin \theta_{i}-a_{j} \sin \theta_{j}, \\
& z=c_{i}-c_{j}
\end{aligned}
$$

$\theta_{\mathrm{i}}, \theta_{\mathrm{j}}, \mathrm{a}_{\mathrm{i}}$ and $\mathrm{a}_{\mathrm{j}}$ are respectively the nodes $\mathrm{i}$ and $\mathrm{j}$ movement directions and velocity.

The authors of [15] present three algorithms (HARP1, HARP2 and HARP3) grouped under the Heading-direction Angles Routing Protocol. In all these algorithms, LET was obtained by applying Equation (1). In order to obtain the angle $(\theta)$, the authors used a different solution from that presented in [14].

The authors of [16] proposed a new approach using a stability function as a selection criterion of the main path based on the computation of the degree of mobility of a node relative to its neighbour.

In [17], the authors proposed a new mechanism for deciding which node should retransmit the received RREQ as a function of the distance of the RREQ transmitter. In this sense, they proposed two protocols: Furthest Candidate Neighbours for Rebroadcasting the RREQ (F-CNRR) and Closest- CNRR (C-CNRR). In case of F-CNRR they allow only the far nodes to rebroadcast the RREQ to gain more coverage area. On the contrary, In case of C-CNRR only the closest nodes to the transmitter of the RREQ will rebroadcast the received RREQ.
The authors of [18] propose the protocol LPBR (Location Prediction-Based Routing). The basic idea behind the LPBR protocol is to include for each node its location and its mobility information in the RREQ packet before transmitting it. When the RREQ packet reaches the destination node, all the collected motion parameters, such as mobility and direction, will be saved in its routing table. This information will be used by the destination node when a route fails to estimate the actual location of the desired node based on the previously collected information.

\section{Energy-Aware Routing Protocols}

The key concept of the Energy-Aware routing protocols is to properly manage the node's energy consumption to extend the network lifetime. In this sense, the Minimum Battery Cost Routing (MBCR) protocol is suggested in [19], where an e2e path is selected based on the RE summation criteria of all nodes participating in the individual path. However, the trouble with such a technique is that it can choose an e2e path including weak residual energy nodes, which can then cause frequent path breaks. To remedy the shortcomings of the MBCR protocol, the Max-Min Battery Cost Routing protocol (MMBCR) selects a path having nodes with a maximum of $\mathrm{RE}$ relative to the other nodes of the network. This approach uses the minimal mobile node RE to evaluate each MMBCR path. Afterwards, the destination node chooses the highest value for each path and sends back the RREP to the source node.

However, the author of [20] introduces the Conditional Maximum Battery Capacity Routing protocol (CMMBCR) that attempts to extend the lifetime of the nodes by selecting only paths containing nodes with a battery power greater than a predefined threshold.

The Authors of [21] succeed to conserve the power of the mobile node by using the uni-cast packet to find any route to destination node rather than using the broadcast message. This technique helps in reducing the consumption of the mobile node and imposes lower overhead.

The Improved-AODV is proposed in [22] to treat selfish nodes in the network, using the remaining power and a new technique that records the nodes acceptance for helping in relay the data. In addition, to extend the network lifetime, IAODV selects network nodes with significant residual energy and therefore a high probability of data transmission.

In [23], a bandwidth-based energy-efficient routing protocol is proposed to save energy and extend the network lifetime. The suggested algorithm measures the RSS and exploits it to evaluate the bandwidth using a specific dB-tobandwidth table. Moreover, this method proposes to use RSS variation to evaluate link lifetimes and predict the amount of data that could be transferred.

In our previous work [24], Fixed-Link Stability and Energy-Aware (F-LSEA) protocol is suggested. In this method, the RREQ message will be forwarded only if it satisfies the link lifetime and the residual energy conditions for the transmitter nodes, according to their fixed thresholds of RE and LLT. 


\section{METHODOLOGY}

In the following, the design of the suggested protocols and their variants will be presented and discussed.

\section{A. Problem Statement}

As discussed earlier, there are two main reasons leading to link breaks: a node dies from depletion of its battery and a node that leaves the coverage area of the radio range of its neighbouring node.

Fig. 1 illustrates the effect of LLT on the network, where there are six nodes including source node "S" and destination node "D". Each link maintains a link lifetime value defining the quality of the link connecting two communicating neighbouring nodes. The source node "S" broadcasts the RREQ to all the nodes of the network. Node 1 and Node 2 will receive this RREQ, and then register the node "S" as the reverse path in their routing table. Then they will rebroadcast the RREQ because it is assumed that "D" does not exist as a valid entry in their routing table. Similarly, node 3 and node 4 receive the RREQ packets respectively from node 1 and node 2 , which will be registered as a reverse path for "S", in the routing table of nodes 3 and 4 .

Then, these last nodes will rebroadcast the RREQs accordingly. Moreover, the node 3 receives and discards the duplicate RREQ received from the node 4 . The RREQ packet sent by node 3 reaches the destination node "D" and finally prepares to reply with the RREP packet.

The route $(\mathrm{D}, 3,1, \mathrm{~S})$ is considered as a reverse path from the destination node " $\mathrm{D}$ " to the source node "S".

According to the lifetime value between nodes D and 3, equal to 5 seconds, the RREP packet sent by $\mathrm{D}$ will successfully reach node 3 . For the same reason, the RREP sent by node 3 will reach node 1 , because the link lifetime between nodes 1 and 3 is equal to 2 seconds.

Unfortunately, the link between nodes 1 and $S$ may be broken due to the weakness of the link (when receiving the RREQ, LLT equals $0.5 \mathrm{sec}$.), even if the node $\mathrm{S}$ receives the RREP packet returned by node 1 . Admittedly, the weakest link will be broken after some transmissions due to the movement and the speed of nodes 1 and $\mathrm{S}$; as the LLT between two nodes depends on their movements and speeds (1).

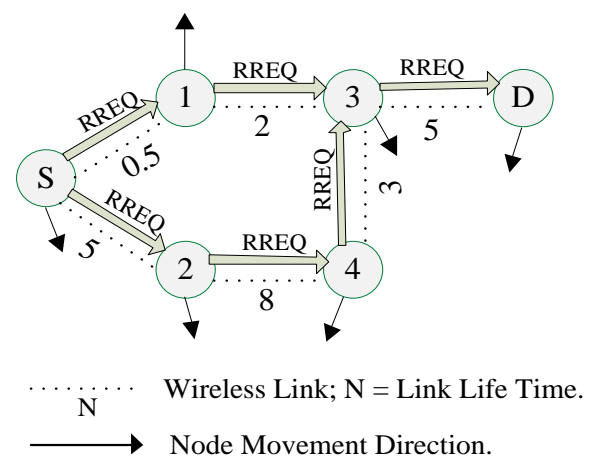

Fig. 1. An example illustrating the effect of Link Lifetime (LLT).

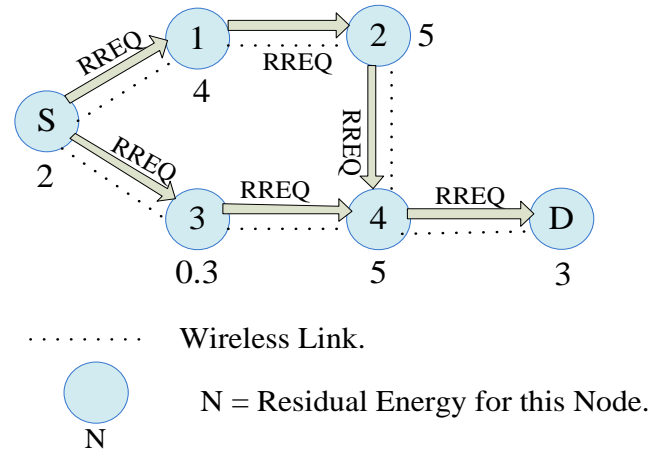

Fig. 2. An example illustrating the effect of Residual Energy (RE).

Similarly, Fig. 2 illustrates the same network as shown in Fig. 1, but involves the nodes RE on the network instead of the LLT. In this case, if the node $\mathrm{S}$ selects the route $(\mathrm{S}, 3,4$, D) to transmit data, the link will be broken after the transmission of some packets due to the low RE of node 3, which is equal to 0.3 .

\section{B. Preliminaries}

In order to enhance the route discovery mechanism, by only allowing nodes that can check specific conditions, two routing protocols have been suggested, namely, 1) F-LSEA; and 2) A-LSEA.

The suggested protocols use the Equation (1) to calculate the link lifetime (LLT). For example, if the LLT value between two nodes in the network equals to 3 , it means that the link connecting them will be broken after 3 seconds. Furthermore, the mobile nodes can easily get their RE.

\section{Fixed-LSEA routing protocol}

The main purpose of this section is to enhance the e2e route discovery mechanism every time a node tries to reach a destination node for which it has no entry in its routing table. As described in the simulation setup (Section IV), the RE and the LLT thresholds have been set to specific values. When using the F-LSEA protocol, if no path has been previously defined between two nodes, the source node broadcasts the RREQ to its neighbouring nodes. Upon receipt of the RREQ, any neighbouring node must verify two necessary conditions before rebroadcasting. The first is to compare its residual energy with the fixed threshold. If it is below the threshold, the current RREQ will be rejected. Otherwise, the node goes to the second necessary condition. The second check is to compare its link lifetime with the fixed threshold that has been pre-defined. If it is below the threshold, the RREQ will be rejected; otherwise, it will be retransmitted. Both conditions must be checked before the neighbouring node transfers the received RREQ.

The proposed F-LSEA protocol aims to achieve efficiency and simplicity. This made it possible to differentiate this protocol from its precedents. In fact, F-LSEA receives a RREQ packet on any node and therefore decides to retransmit or not the RREQ according to its RE and LLT.

On the other hand, in previous protocols such as [19]-[21], all the nodes rebroadcast all the received RREQ packets, and enable the destination node to choose a route according to the 
received RREQ packet. This route includes nodes with acceptable LLT and a high RE level, where LLT and RE are used as metrics respectively. Thus, concerning the F-LSEA protocol, the following fundamental question was raised: why does a node have to transmit a RREQ when its LLT with the sender of the RREQ is about to be broken and the reply can never reach the RREQ transmitter?

Moreover, redundant RREQs packets cause more overhead when only one route will be selected at the end. Unlike the previous work, the F-LSEA protocol removes most of the redundant paths from the beginning by choosing the best paths.

Fig. 3 illustrates an ad-hoc network topology to better understand the F-LSEA protocol. The network consists of five nodes, where each node is identified by an address (number inside the circle). The value under each node represents the $\mathrm{RE}$, while the number below the links defines the respective LLTs. In this example, we define the value of the RE and LLT thresholds equal to 3 . The source node " $\mathrm{S}$ " wishes to communicate with the destination node " $\mathrm{D}$ " by using other intermediate nodes (node 1 , node 2 and node 3 ) in an ad-hoc network.

Suppose that the source node "S" does not have any route in its routing table to reach the destination node " $\mathrm{D}$ ". Then, the source node will broadcast a RREQ packet to all its neighbour nodes. For the classic AODV, the receiving nodes (1, 2 and 3 in our case) will rebroadcast the RREQ packet, if there is no valid route that exists to reach the destination.

In the case of our F-LSEA protocol, on the one hand, node 1 verifies the first necessary condition with respect to its LLT value with the source node "S" (knowing that the LLT threshold $=3$ seconds). On the other hand, if the first condition is satisfied, it checks the second energy-related condition (threshold $\mathrm{RE}=3$ Joule). If the node's RE value is insufficient (less than threshold), the node rejects the received RREQ packet.

The same steps will be applied to the other intermediate nodes (node 2 and 3 ). The node 3 receives the packet RREQ and realizes that it's RE level is greater than the threshold 3. Only, by checking the second condition, the node 3 detects that its link lifetime with the source node " $\mathrm{S}$ " is low (LLT = 2 $<3)$, so it rejects the RREQ packet.

In this diagram of the example (Fig. 3), only the node 2 can retransmit the received RREQ packet because it satisfies the two conditions relative to RE and LLT.

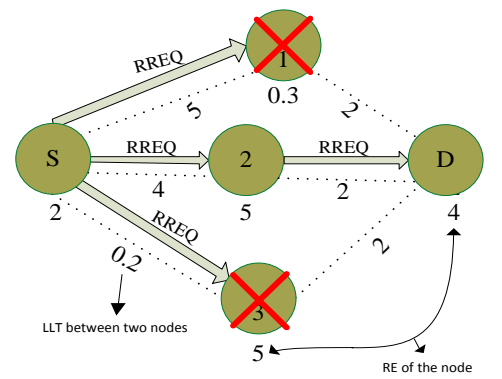

Fig. 3. Operating scenario of the F-LSEA protocol.
The decision of each node receiving the RREQ packet relied on the following algorithm (Algorithm 1).

\section{Algorithm 1: F-LSEA}

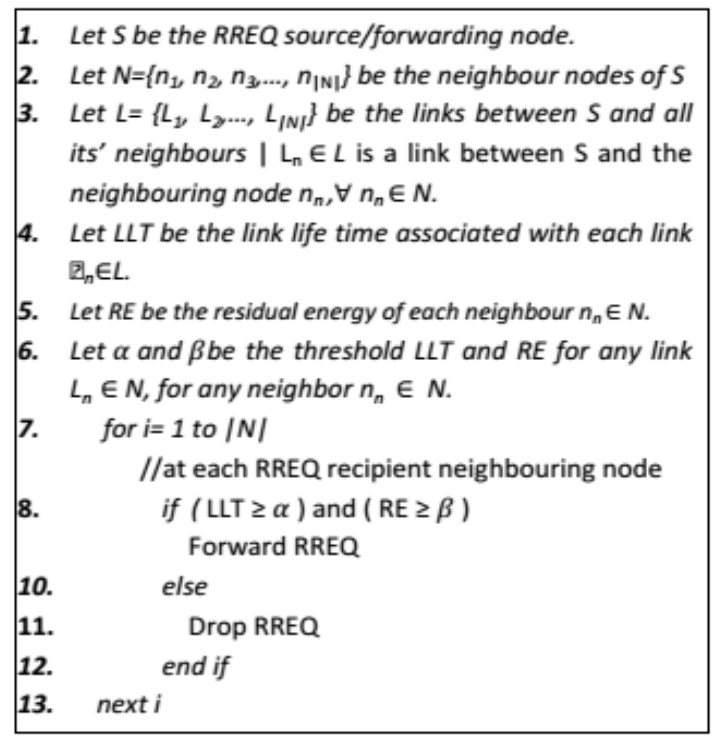

As can be observed in Algorithm 1, this verifies for each node and at each reception of RREQ, whether the LLT and the RE satisfy the requirements of the predefined thresholds $(\alpha$ and $\beta$ ). At line 9 of the algorithm, the RREQ is forwarded if both conditions are satisfied, otherwise it will be rejected (line 11). The same actions are also illustrated using the flowchart in Fig. 4.

\section{Average-LSEA routing protocol}

The stability of the link at each hop is guaranteed by the FLSEA algorithm because the decision of forwarding/discarding the RREQ is taken at each receiver node upon receiving the RREQ based on the LLT relative to the RREQ sender and its RE. Nevertheless, the forwarding or discarding the RREQs is entirely based on a specific node RE and LLT thresholds. The fixed thresholds, on the one hand, and the decision-making of the receiver, on the other hand, are not sufficiently flexible under these conditions of fixed thresholds.

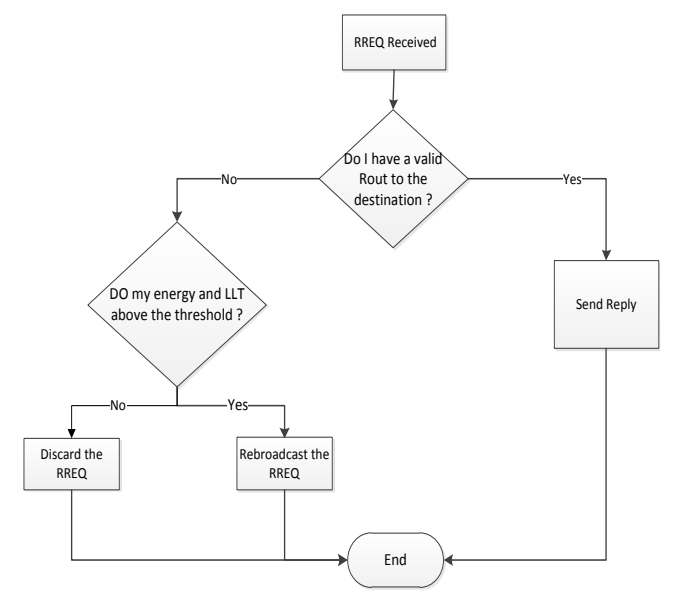

Fig. 4. Flow Chart illustration for the F-LSEA algorithm for each node. 


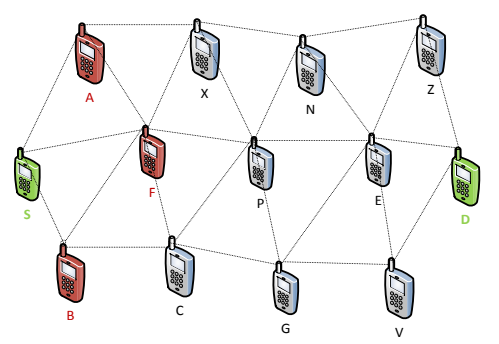

Fig. 5. Example of an isolated node.

This inflexibility occurs for two principal reasons: First of all, one can fall in the case where all the neighbouring nodes of the receiving node have their RE and LLT lower than the predefined thresholds.

As shown in Fig. 5, the isolation of the receiving node occurs due to the inability of neighbouring nodes to rebroadcast the received RREQ packet to the next hop.

As shown in the example of Fig. 5, the source node "S" sends a RREQ asking for " $D$ " as destination. After receiving the RREQ at nodes A, B and F, they check if there are routes to reach the destination node "D". If yes, a RREP packet will be send to the source node "S". Otherwise, they will proceed with the decision-making process to determine whether or not to forward the RREQ packet to their neighbours.

In such cases, the $\mathrm{B}, \mathrm{F}$ and $\mathrm{A}$ nodes verify their residual energy and link lifetime parameters with the source node "S", from which the RREQ has been received. Suppose that one of the RE or LLT parameter of all neighbouring nodes of $\mathrm{S}$ is less than the predefined threshold. In that case, there is no possible scenario for node "S" to propagate its RREQ across the network. This issue persists in the suggested Fixed-LSEA protocol.

Therefore, the Average-LSEA routing protocol is suggested in order to overcome this deficiency of FixedLSEA.

Unlike the F-LSEA protocol, the A-LSEA is particularly based on the average values of the RE and LLT parameters, which can be calculated using the following methods:

- A node periodically sends a "hello" message to its neighbour nodes. Each node receiving this message responds with a modified "hello" message including its $\mathrm{RE}$ and its coordinates (x, y), as illustrated by Fig. 6. In this way, each node can sum the RE of its neighbouring nodes to obtain the average RE (REavg), using (2).

Adding the coordinates (x,y) of the node to the "hello" message allows the receiving node to calculate its LLT with that node (Sender of the Hello).

- Each node in the network can receive LLTs from all its neighbour nodes. In order to compute the mean LLT (LLTavg), the considered node sums its LLT value with the LLT values of the other neighbouring nodes and divides the obtained total by the number of neighbours, using (3).

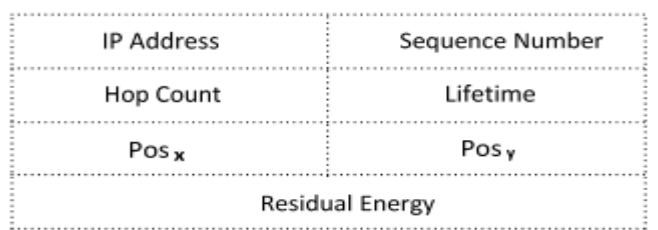

Fig. 6. Modified "hello" message including the node RE and its coordinates $(\mathrm{x}, \mathrm{y})$.

Consider the A-LSEA protocol, when receiving RREQ by any node; it consults its routing table searching a route for the current request. If the result of the query is negative, it means that there is no existing route in its routing table; it calculates the average values $R_{\text {avg }}$ and $L_{L T}$ avg of all its neighbours. Then, it compares its LLT and RE parameters with those of the calculated averages: If the LLT and the RE are respectively greater than or equal to LLTavg and $\mathrm{RE}_{\mathrm{avg}}$, the node forwards the RREQ. Otherwise, it will be discarded.

As described in Fig. 5, the source node "S" looks for an e2e path to reach the destination node " $D$ ", obviously; "S" broadcasts the RREQ to all its neighbouring nodes. First of all, it should be noticed that source node $S$ is the origin of the RREQ packet and that it will always broadcasts the RREQ to its neighbours. So the RREQ originator node (that is to say the source node of the e2e path) is eliminated from the A-LSEA protocol. Therefore, the source node will always follow the path discovery mechanism of reactive routing protocols, such as AODV. All other network nodes forward the RREQ packet in accordance to A-LSEA protocol as described below.

\section{Algorithm 2: A-LSEA}

\section{Input: Local information on neighbours' RE and LUT.} Output: A stable end-to-end routing path.

1. Let $S$ be the RREQ source/forwarding node.

2. Let $N=\left\{n_{1}, n_{2}, n_{3} \ldots, n_{|N|}\right\}$ be the neighbour nodes of $S$

3. Let $L=\left\{\lambda_{1}, \lambda_{2} \ldots, \lambda_{|N|}\right\}$ be the links between $S$ and all its neighbours $\mid \lambda_{n} \in L$ is a link between $S$ and the neighbouring node $n_{n v} \forall n_{n} \in N$.

4. Let $L L T_{A v g}$ be the averaged link lifetime associated with all neighbouring node links and the set threshold.

5. Let $R E_{A v g}$ be the averaged residual energy of all neighbouring nodes, including source $\mathrm{S}$.

6. for $i=1$ to $|N|$ //at each RREQ recipient neighbouring node

7. if ( $L L T \geq L L T_{\text {Avg }}$ ) and ( $R E \geq R E_{\text {Avg }}$ )

8. Forward RREQ to all neighbours.

9. else

10. Drop RREQ

11. end if

12. next $i$ 
Suppose " $F$ " is the candidate node that decides about the routing of the RREQ packet according to the A-LSEA algorithm. To generalize:

Let $\mathrm{N}=\{\mathrm{N} 1, \mathrm{~N} 2, \mathrm{~N} 3 \ldots|\mathrm{N}|\}$ the set of neighbouring nodes of $\mathrm{F}$.

Let $T=\{N\} U$ S represent the collection of neighbouring nodes with the source/forwarding node, and let $R E=\left\{R E_{1}\right.$, $\left.\mathrm{RE}_{2}, \mathrm{RE}_{3}, \ldots, \mathrm{RE}_{|\mathrm{N}|}\right\}$ the REs of the $\mathrm{N}$ neighbouring nodes.

The average RE of all neighbouring nodes is calculated using (2):

$$
R E_{A v g}=\sum_{i=1}^{|T|} \frac{R E_{i}}{|T|}
$$

Likewise, let $\left(\mathrm{LLT}_{1}, \mathrm{LLT}_{2}, \ldots, \mathrm{LLT}_{|\mathrm{N}|}\right)$ the LLTs between the node " $F$ " and its neighbouring nodes. The average LLT is obtained using the following (3):

$\mathrm{LLT}_{\text {Avg }}=\sum_{\mathrm{i}=1}^{|\mathrm{N}|} \frac{\mathrm{LLT}_{\mathrm{i}}}{|\mathrm{N}|}$

As described in Fig. 5, if "F" receives the RREQ packet from node " $\mathrm{S}$ " and LLT $(\mathrm{S}-\mathrm{F})$ represents the LLT between these two nodes. The node " $F$ " will then compare its residual energy and $\mathrm{LLT}_{(\mathrm{S}-\mathrm{F})}$ with respectively the average residual energy and $\mathrm{LLT}_{\mathrm{Avg}}$, of its neighbours. If both parameters residual energy and $\operatorname{LLT}_{(\mathrm{S}-\mathrm{F})}$ of the node "F" are, respectively, greater than or equal to the calculated $\mathrm{RE}_{\mathrm{Avg}}$ and $\mathrm{LLT}_{\mathrm{Avg}}$, (3) and (2), the node " $F$ " rebroadcasts the RREQ packet otherwise, it will be rejected (Algorithm 2).

\section{PERFORMANCE EVAlUATION}

The NS2 network simulator [25] is used to evaluate the performance of the suggested A-LSEA protocol. Simulation parameters, scenarios, performance measures and results are presented and discussed in the following sections.

\section{A. Simulation Environment}

The configuration of the MAC layer within the implemented simulation runs on the IEEE 802.11 Distributed Coordination Function (DCF) [26]. The transmission range of any node was fixed at 250 meter and the bandwidth set at 2 Mbps. To gather the result a different scenario was carried out using 100 nodes to simulate the network, these node disseminated in area of 600 meter $^{2}$. The mobility of the nodes was simulated using Random Waypoint [27]. In this model, each node begins its movement with a randomly selected velocity, chosen within the interval $[5 \mathrm{~m} / \mathrm{s}, 30 \mathrm{~m} / \mathrm{s}]$, from its current location to a random location. The simulation time of each scenario lasts 600 seconds. All tests used the same fixed packet size of 1 Kilobyte using Constant Bit Rate (CBR) as the flow type, generated at a constant interval rate of 4 packets per second. Also, 15 flows have been scheduled and configured to randomly select a source node and a destination node for the simulation period.

Finally, the LLT was set at 2 seconds and the initial values of the REs ranged from 1 to 4 Joules.

\section{B. Simulations Results and Discussion}

In the following subsections, three routing protocols that are A-LSEA, F-LSEA and AODV have been compared and analyzed for their performance in the wireless network.

\section{1) Packet Delivery Ratio}

As shown in Fig. 7, the combined effects of RE and LLT affect the data delivery ratio. Indeed, the curve shape provided by the suggested protocols (F-LSEA and A-LSEA) both give a better average delivery ratio than that of the AODV. This is mainly due to the e2e routes returned by F-LSEA and ALSEA protocols, which have a longer route lifetime and are more stable than AODV. These protocols regard paths with nodes having the highest RE levels and an acceptable LLT, by performing localized and distributed algorithms represented respectively by Algorithm 1 and 2.

However in the case of the AODV protocol, network nodes are unable to capture the REs and LLTs of their neighbours, and are therefore unable to discern the best from the worst links. Thereby, AODV blindly scatters the RREQ packets in the network and can therefore provide paths with faulty individual links, resulting in greater packet loss. In addition, A-LSEA protocol outperforms F-LSEA due to the flexibility of the average values of LLT and RE with respect to the state of the nodes and the state of the network.

\section{2) Network Life Time}

Consider the F-LSEA protocol, Fig. 8 shows the lifetime of the network, which increases with the increase of the energy threshold. Increasing the energy threshold (from 1 to 4 ) can prevent node whose energy is below this level from forwarding the RREQ. This prevents many nodes from forwarding the RREQ and, as a result, to save their energy and thus improve the network lifetime. Moreover, the simultaneous exchange of several RREQs actually causes the premature death of the nodes, and consequently, they can no longer belong to the network. The elimination of nodes in the F-LSEA protocol, however, saves energy of the network by saving the energy of the nodes, preventing them from transmitting and receiving RREQs. In addition, the choice of more stable paths when using the F-LSEA protocol generates little overhead for path maintenance and therefore less energy consumption.

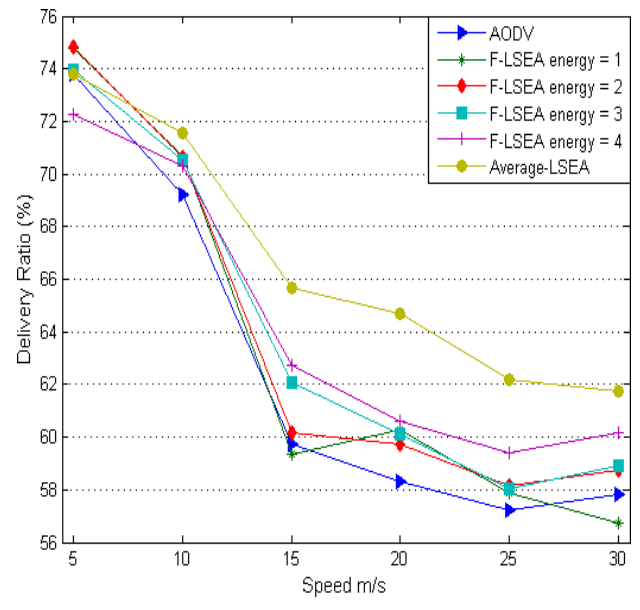

Fig. 7. Delivery Ratio vs. Speed. 


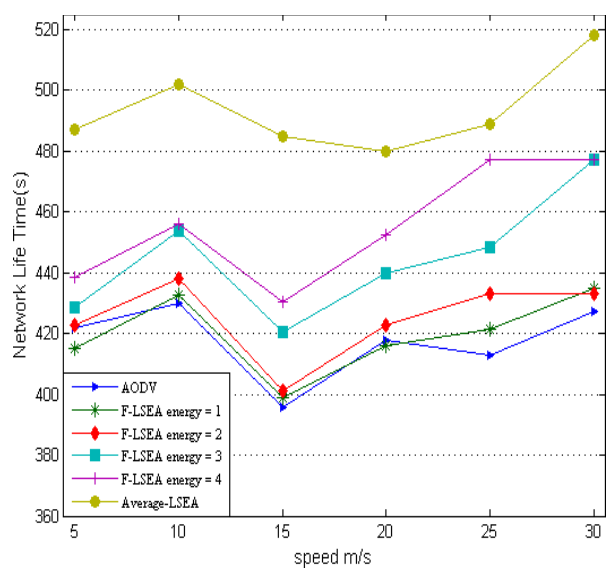

Fig. 8. Network lifetime vs. Speed.

Some RREQ packets may be considered useless in the case where the path created by these RREQ packets is broken, and thus the node becomes unreachable because of the poor link quality or low power.

Reduce the number of useless RREQ packets sent over the network allows the nodes to save energy and thereby increase the network lifetime. The A-LSEA algorithm performs better than the F-LSEA (LLT $=2$ and RE $=1,2,3$ or 4) because of the flexible average thresholds, whereas for F-LSEA, the threshold values are fixed. This flexibility in A-LSEA protocol results in more stable links compared to F-LSEA, and thus a better total network lifetime.

\section{3) End-To-End Delay}

As can be seen in Fig. 9, the average packet delay observed in the case of AODV is higher than the protocols suggested in the majority of cases. However, in some cases, the average delay of the suggested protocols is higher.

The main reason is that the suggested algorithms choose a link based on its quality and the remaining battery lifetime. As the e2e path returned by the suggested protocols may have more hops compared to AODV, the packets may experience further delays due to more transmission and queues along the path.

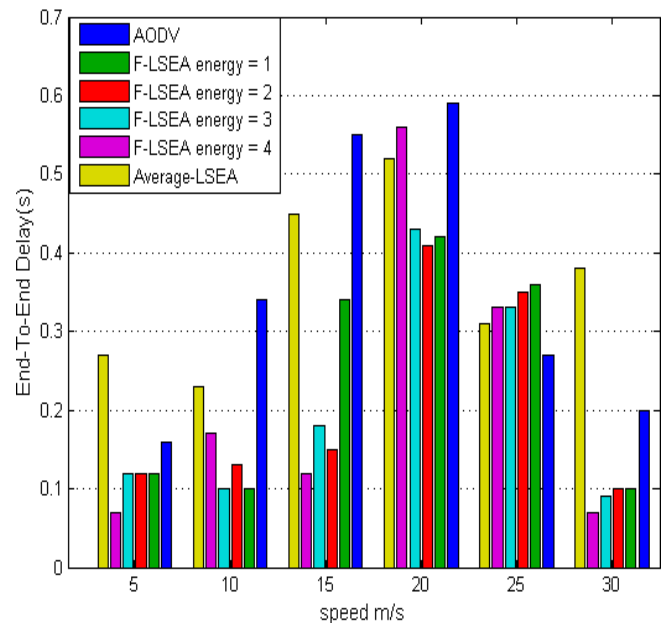

Fig. 9. e2e Delay vs. Speed.

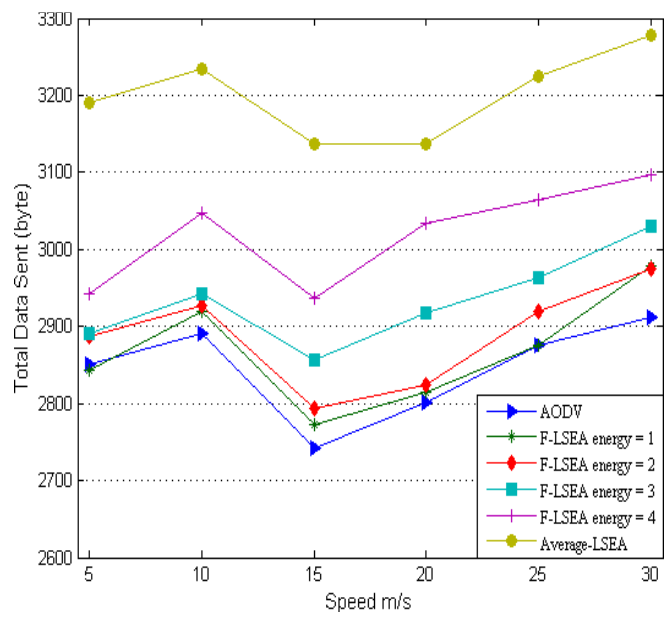

Fig. 10. Total Data Sent vs. Speed.

\section{4) Total Data Sent}

As shown in Fig. 10, which illustrates a comparison between the suggested algorithms and AODV in terms of data sent, A-LSEA performs significantly better than the F-LSEA and AODV protocols.

The main reason is that for the path discovery phase, ALSEA algorithm uses an appropriate mechanism to estimate link quality and RE, by adopting their mean values. This more stable path choice result in less link breaks, and therefore the total amount of data sent is higher than in the case of other algorithms. In addition, F-LSEA performs better (for RE varying from 1 and 4) than AODV because of the prevention of many nodes to transmitting the RREQ. Likewise, increasing the RE threshold causes more RREQ packet drops.

\section{5) Total Received Data}

All the nodes forming the network consume power during the transmission and reception of the data and, simultaneously, the power consumption increases as the distance separating the transmitter and the receiver increases. For these reasons, A-LSEA performs significantly better than F-LSEA and AODV, as illustrated by Fig. 11.

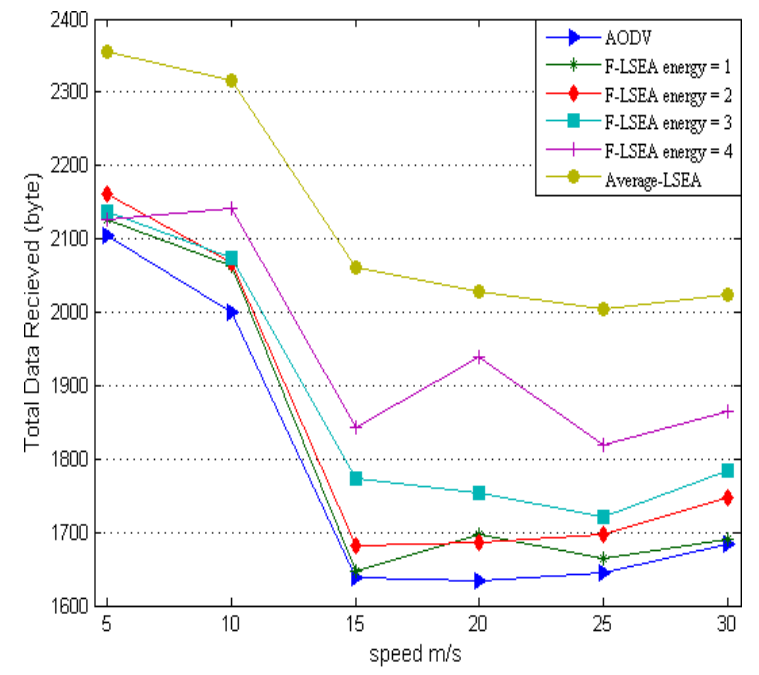

Fig. 11. Total Data bytes Received vs. Speed. 
Indeed, the A-LSEA protocol returns the e2e paths that are able to maintain the stability of the route and to provide moderate distances among the nodes; likewise, the main factors affecting the calculation of LLT and RE between two nodes among the nodes that are concerned by the calculation of the route. Clearly, the total amount of data bytes received decreases for all observed protocols as the speed of the nodes increases. Moreover, for the same simulation parameters, the F-LSEA algorithm (for RE varying from 1 to 4) outperforms AODV in terms of the total amount of data bytes received. As shown in Fig. 11, raising the fixed threshold results in lower RREQs meeting the necessary conditions (fewer RREQ packets sent results in fewer RREQ packets received), which directly impacts the total number of received RREQ packets, leading to the effective reception of more data.

\section{6) Total Data Drop}

This section compares the suggested algorithms against AODV in terms of total data loss. As illustrated in Fig. 12, the A-LSEA protocol has a significant low data loss rate compared to those reported by F-LSEA and AODV protocols. As mentioned earlier, this is due to more stability of paths provided by A-LSEA protocol and therefore, the network will experience less congestion.

Indeed, in the case where the network is congested, it is very likely that the nodes reject more packets than in the case of a low traffic network. In addition, potential interference among nodes during data transmission, collisions, and long queues have the greatest impact on dropping data packets. Furthermore, a very slight variation in the A-LSEA behavior can be reported with respect to F-LSEA and AODV, as the speed of the node increases. This is because A-LSEA generates less RREQ across the network, which results in more channel free time, shorter queues, and fewer collisions. On the one hand, the A-LSEA protocol differs from the AODV and F-LSEA protocols in that it provides more stable routes. These routes are more likely to last long before launching a new path discovery instance, thus reducing overhead on the network. On the other hand, the paths established by the AODV protocol does not last long enough and it is therefore inevitable to search for a new path in a short period of time, which leads to more overhead on the network. Correspondingly, for the F-LSEA protocol (for RE varying from 1 to 4), an already established route will only last for the time set by the predefined threshold before initiating the discovery process of a new path, resulting in more overhead.

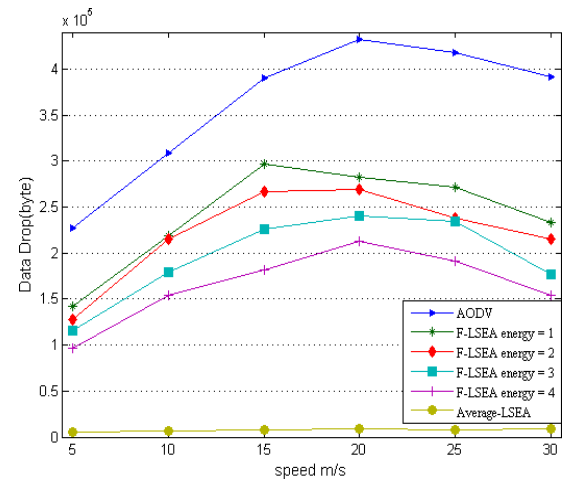

Fig. 12. Total Data Drop vs. Speed.

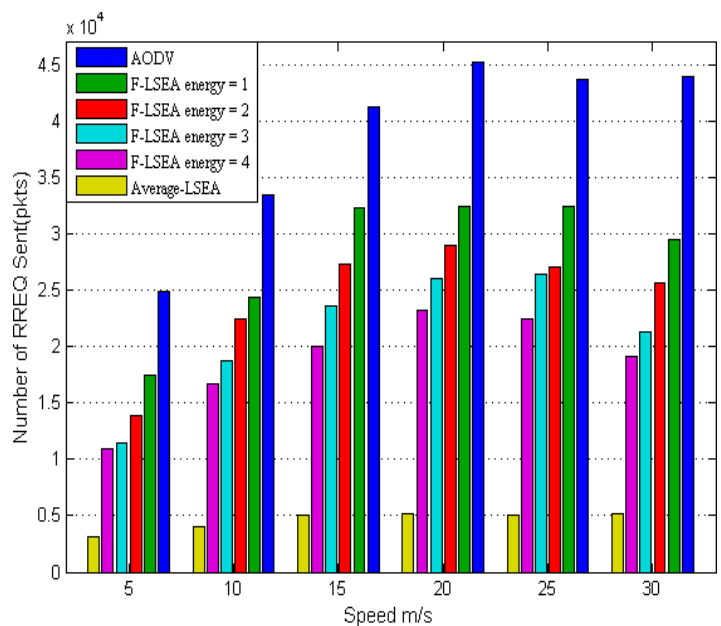

Fig. 13. RREQ Sent vs. Speed.

It should be noticed that during the increase of the predefined threshold, for the F-LSEA algorithm, the overhead decreases because there is less retransmission of RREQ packets.

\section{7) Number of RREQs sent}

As can be seen in Fig. 13, the number of RREQs sent over the network has a considerable effect on the performance of all the routing protocols considered during this work. Indeed, raising the number of RREQ packets circulating on the network leads to more heavily loaded communication. Moreover, nodes dissipate more power when sending or receiving RREQ packets, which impacts the network lifetime, overhead, the effective data bytes sent, the effective data bytes received and the delivery rate of the effective data sent.

It is also noticed that A-LSEA outperforms the F-LSEA (for RE varying from 1 to 4) and AODV methods. Consequently, the fixed RE threshold for the F-LSEA algorithm plays an important role in reducing the number of the RREQ packets retransmitted over the network; decreasing the fixed threshold value increases RREQ packets routing and vice versa.

\section{8) Average Throughput}

A comparison of the average throughput of the studied protocols is shown in Fig. 14.

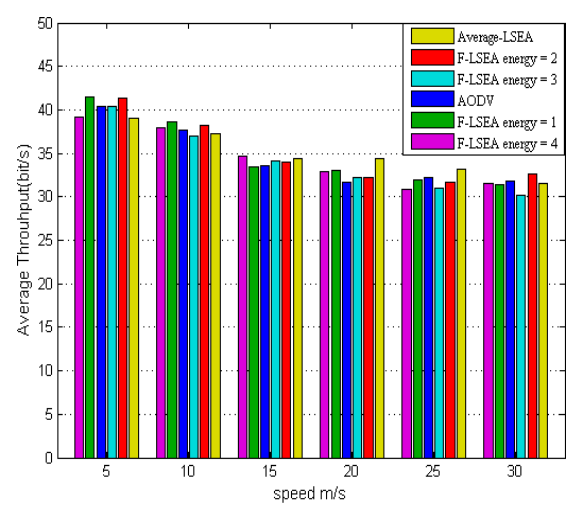

Fig. 14. Throughput vs. Speed. 
It is noticed that the throughput of all protocols decreases with the node speed increases. This is obviously due to the fact that the mobility of the nodes favors the breaks of connections and thus leads to more re-initialization of the e2e routes, which results in a reduction of the flow.

Comparatively, the average throughput remains relatively the same in the three considered routing protocols. The suggested schemes have therefore improved the other metrics while maintaining convergent average throughputs.

\section{CONCLUSION AND FUTURE WORK}

In reactive routing protocols, the route discovery process can consume a lot of network resources due to the dissemination of RREQs to find the path to a destination node. In addition, because of the mobile nature of the nodes in MANETs, stable path selection is extremely important. This article suggests two protocols for dealing with the flood phenomenon that exists in the reactive routing of MANETs. In suggested solutions, only specific nodes are allowed to forward the received RREQs. On the one hand, the decision to include nodes in an e2e path is based on their residual energies (RE). On the other hand, the proposed protocols guarantee stable paths by considering link lifetime (LLT) between two nodes.

Future work consists in finding optimal value for the LLT and the RE with respect to the decision to rebroadcast the received RREQ packet or not, rather than using a fixed or average values threshold.

\section{ACKNOWLEDGEMENT}

Financial support for this study was provided by deanship of Scientific Research, Northern Border University under grant no. (7167-SCI-2017-2-7-F).

\section{REFERENCES}

[1] Garg, N., Aswal, K., \& Dobhal, D. C. (2012). A review of routing protocols in mobile ad hoc networks. International Journal of Information Technology, 5(1), 177-180.

[2] Chlamtac, I., Conti, M., \& Liu, J. J. N. (2003). Mobile ad hoc networking: imperatives and challenges. Ad hoc networks, 1(1), 13-64.

[3] Bheemalingaiah, M., Naidu, M. M., Rao, D. S., \& Vishvapathi, P. (2017, January). Performance Analysis of Power-Aware Node-Disjoint Multipath Source Routing in Mobile Ad Hoc Networks. In Advance Computing Conference (IACC), 2017 IEEE 7th International (pp. 361371). IEEE.

[4] Perkins, C. E., Ratliff, S., Dowdell, J., Steenbrink, L., \& Mercieca, V. (2016). Ad Hoc On-demand Distance Vector Version 2 (AODVv2) Routing. Internet Draft (Standards Track), Mobile Ad hoc Networks Working Group. Available at http://tools. ietf. org/html/draft-ietf-manetaodvv2-13.

[5] Salem, A. O. A., Samara, G., \& Alhmiedat, T. (2017). Performance analysis of dynamic source routing protocol. arXiv preprint arXiv: 1712.04622 .

[6] Ouyang, R. W., Wong, A. K. S., \& Lea, C. T. (2010). Received signal strength-based wireless localization via semidefinite programming: Noncooperative and cooperative schemes. IEEE Transactions on Vehicular Technology, 59(3), 1307-1318.

[7] Bolla, R., Bruschi, R., Davoli, F., \& Cucchietti, F. (2011). Energy efficiency in the future internet: A survey of existing approaches and trends in energy-aware fixed network infrastructures. IEEE Communications Surveys \& Tutorials, 13(2), 223-244.

[8] Athanasiou, G., Korakis, T., Ercetin, O., \& Tassiulas, L. (2009). A cross-layer framework for association control in wireless mesh networks. IEEE Transactions on Mobile Computing, 8(1), 65-80.

[9] Dube, R., Rais, C. D., Wang, K. Y., \& Tripathi, S. K. (1997). Signal stability-based adaptive routing (SSA) for ad hoc mobile networks. IEEE Personal communications, 4(1), 36-45.

[10] Wang, S. Y., Liu, J. Y., Huang, C. C., Kao, M. Y., \& Li, Y. H. (2005, March). Signal strength-based routing protocol for mobile ad hoc networks. In Advanced Information Networking and Applications, 2005. AINA 2005. 19th International Conference on (Vol. 2, pp. 17-20). IEEE.

[11] Huang, D. W., Lin, P., \& Gan, C. H. (2008). Design and performance study for a mobility management mechanism (WMM) using location cache for wireless mesh networks. IEEE Transactions on Mobile Computing, 7(5), 546-556.

[12] Jacquet, P., Muhlethaler, P., Clausen, T., Laouiti, A., Qayyum, A., \& Viennot, L. (2001). Optimized link state routing protocol for ad hoc networks. In Multi Topic Conference, 2001. IEEE INMIC 2001. Technology for the 21st Century. Proceedings. IEEE International (pp. 62-68). IEEE.

[13] Misra, P., \& Enge, P. (2006). Global Positioning System: signals, measurements and performance second edition. Massachusetts: GangaJamuna Press.

[14] Su, W., Lee, S. J., \& Gerla, M. (2000). Mobility prediction in wireless networks. In MILCOM 2000. 21st Century Military Communications Conference Proceedings (Vol. 1, pp. 491-495). IEEE.

[15] Gerharz, M., de Waal, C., Frank, M., \& Martini, P. (2002, November). Link stability in mobile wireless ad hoc networks. In Local Computer Networks, 2002. Proceedings. LCN 2002. 27th Annual IEEE Conference on (pp. 30-39). IEEE.

[16] Moussaoui, A., Semchedine, F., \& Boukerram, A. (2014). A link-state QoS routing protocol based on link stability for Mobile Ad hoc Networks. Journal of Network and Computer Applications, 39, 117-125.

[17] Hamad, S., Belhaj, S., \& Muslam, M. M. (2017). Smart Selection of Candidate Neighbors for Efficient Route Discovery in MANETs. Journal of Applied Sciences, 17, 126-134.

[18] Meghanathan, N. (2008). A location prediction-based reactive routing protocol to minimize the number of route discoveries and hop count per path in mobile ad hoc networks. The Computer Journal, 52(4), 461-482.

[19] Singh, S., Woo, M., \& Raghavendra, C. S. (1998, October). Poweraware routing in mobile ad hoc networks. In Proceedings of the 4th annual ACM/IEEE international conference on Mobile computing and networking (pp. 181-190). ACM

[20] Toh, C. K., Cobb, H., \& Scott, D. A. (2001). Performance evaluation of battery-life-aware routing schemes for wireless ad hoc networks. In Communications, 2001. ICC 2001. IEEE International Conference on (Vol. 9, pp. 2824-2829). IEEE

[21] Gelenbe, E., \& Lent, R. (2004). Power-aware ad hoc cognitive packet networks. Ad Hoc Networks, 2(3), 205-216.

[22] Chen, C. W., Weng, C. C., \& Kuo, Y. C. (2010). Signal strength based routing for power saving in mobile ad hoc networks. Journal of Systems and Software, 83(8), 1373-1386

[23] Xu, Y., Heidemann, J., \& Estrin, D. (2001, July). Geography-informed energy conservation for ad hoc routing. In Proceedings of the 7th annual international conference on Mobile computing and networking (pp. 7084). ACM.

[24] Hamad, S., Noureddine, H., \& Al-Raweshidy, H. (2011, October). Link stability and energy aware for reactive routing protocol in mobile ad hoc network. In Proceedings of the 9th ACM international symposium on Mobility management and wireless access (pp. 195-198). ACM.

[25] Fall, K., \& Varadhan, K. (2007). The network simulator (ns-2). URL: http://www. isi. edu/nsnam/ns. Last visit December 2017.

[26] Bianchi, G. (2000). Performance analysis of the IEEE 802.11 distributed coordination function. IEEE Journal on selected areas in communications, 18(3), 535-547.

[27] Bettstetter, C., Hartenstein, H., \& Pérez-Costa, X. (2004). Stochastic properties of the random waypoint mobility model. Wireless Networks, $10(5), 555-567$. 